\title{
High hydrostatic pressure food processing: An overview
}

\section{Rohit Sharma and Khursheed Alam Khan}

See end of the Paper for authors' affiliation

Correspondence to :

Rohit Sharma Department of Processing and Food Engineering, Punjab Agricultural University, Ludhiana (Punjab) India Email : rohitsharma@pau.edu
- Abstract : High hydrostatic pressure processing (HHPP or HPP or HHP or Pascalization) is a non-thermal food preservation and processing technology that can inactivate food borne pathogens, spoilage micro-organisms and unfavourable enzymes without significantly altering organoleptic properties and nutritional value of foods. The pressure transmission is uniform and quasi-instantaneous during HHP regardless of the size and geometry of food, rendering the technology more effective and energy efficient. HPP utilizes intense pressure (about 400-600 MPa or $58,000-87,000 \mathrm{psi})$ at chilled or mild process temperatures $\left(<45^{\circ} \mathrm{C}\right)$. Pressure treatment can be used to process both liquid and high-moisture-content solid foods. Although lethal to microorganisms, pressure treatment does not break covalent bonds and has a minimal effect on food chemistry. Thus, HPP provides a means for retaining food quality while avoiding the need for excessive thermal treatments or chemical preservatives. In addition to lengthening the shelf-life of food products, HHP can modify functional properties of components such as proteins, which in turn can lead to the development of new products. In light of these reasons, the use of HHP for processing food has resurged with the industry's renewed interest in its application. Within the last decade, a number of companies have introduced commercial grade high pressure systems thus providing food processors an opportunity to preserve foods and offering a process of choice for applications where alternative processing methods would adversely impact product quality.

- Key words : Food preservation, Microbial inactivation, New technologies, Non-thermal processes

- How to cite this paper : Sharma, Rohit and Khan, Khursheed Alam (2018). High Hydrostatic Pressure Food Processing: An Overview. Internat. J. Agric. Engg., 11(Sp. Issue) : 70-75, DOI: 10.15740/HAS/IJAE/11.Sp. Issue/70-75. 